\title{
Auto-detection interpretation model for horizontal oil wells using pressure transient responses
}

\author{
Seyedeh Raha Moosavi ${ }^{1}$, Behzad Vaferi², David A.Wood ${ }^{3 \oplus *}$ \\ ${ }^{1}$ Department of Chemical and Petroleum Engineering, Shiraz University, Shiraz, Iran \\ ${ }^{2}$ Department of Chemical Engineering, Shiraz Branch, Islamic Azad University, Shiraz, Iran \\ ${ }^{3}$ DWA Energy Limited, Lincoln, United Kingdom
}

\section{Keywords:}

Horizontal well pressure

pressure transient analysis

reservoir pressure prediction

noisy pressure transient data

multilayer perceptron neural network

Cited as:

Moosavi, S.R., Vaferi, B., Wood, D.A. Auto-detection interpretation model for horizontal oil wells using pressure transient responses. Advances in

Geo-Energy Research, 2020, 4(3):

305-316, doi:

10.10.46690/ager.2020.03.08.

\begin{abstract}
:
Directional drilling is an excellent option to extend the limited reservoir reach and contact offered by vertical wells. Pressure transient responses (PTR) of horizontal wells provide key information about the reservoirs drilled. In this study multilayer perceptron (MLP) neural networks are used to correctly identify reservoir models from pressure derivative curves derived from horizontal wells. To this end, 2560 pressure derivative curves for six distinct reservoir models are generated and used to design a machine-learning classifier. A single hidden layer MLP network with 5 neurons, trained with a scaled conjugate gradient algorithm, is selected as the best classifier. This smart classifier provides total classification accuracy of $98.3 \%$, mean square error of 0.00725 , and coefficient of determination of 0.97332 over the whole dataset. Performance accuracy of the proposed classifier is verified with real field data, synthetically generated noisy PTR, and some signals outside the range initially assessed by the training plus testing data subsets. The developed network can correctly identify the reservoir-flow model with a probability of close to 0.9 . The novelty of this work is that it employs a large dataset of horizontal (not vertical) well tests applied to six reservoir-flow models and includes noisy data to train and verify a neural network model to reliably achieve a high-level of prediction accuracy.
\end{abstract}

\section{Introduction}

Horizontal and vertical wells behave quite differently in terms of the flow regimes they experience related to the reservoirs they drill. Horizontal wells are typically drilled in highangle direction $\left(80^{\circ}\right.$ to $90^{\circ}$ from vertical) that begin as with vertical trajectories near the surface but become horizontal at depth in the target zones. They are drilled to improve reservoir performance by penetrating producing formations with long wellbore sections. Furthermore, reducing gas and water coning in oil reservoirs, reducing the pressure drop and fluid velocity around the wellbore, minimizing sand/proppant production, and increasing production rate are some of the advantages of horizontal wells. These benefits typically lead to higher recovery from horizontal wells relative to the vertical wells.

Since the 1930's, bottom-hole pressures have been assessed in wellbores to enhance petroleum production and recovery by providing more insight to fluid flow in sub-surface reser- voirs. At the outset, reservoir pressure values were attained by simply measuring fluid levels. One-time-reading pressure bombs, maximum-indicating and/or maximum-recording pressure gauges were gradually introduced over time. The early pressure gauges lacked desirable reliability and accuracy compared to the downhole pressure gauges used today. This made the interpretation of bottom-hole pressures unreliable. As instrumentation technologies have advanced, and better production tools developed, it has become possible to conduct bottom-hole (reservoir) pressure analysis with more confidence. The technology advances in accurate down-hole pressure recording has made well-test interpretation possible and reliable, focusing on both build-up and drawdown analysis in complex well trajectories and heterogeneous reservoirs (Meng et al., 2020; Zhao and Du, 2020).

Pressure transient responses of petroleum reservoirs are among the most important sources of information for pre-

\section{\begin{tabular}{c|} 
Yandy \\
Scientific \\
Press
\end{tabular}}

${ }^{*}$ Corresponding author.

E-mail address: r.moosavi_820@yahoo.com (S.R. Moosavi); behzad.vaferi@gmail.com (B. Vaferi);

dw@dwasolutions.com (D.A. Wood).

2207-9963 (C) The Author(s) 2020.

Received June 26, 2020; revised July 12, 2020; accepted July 12, 2020; available online July 14, 2020. 
dicting well and reservoir properties and likely production performance (Vaferi and Eslamloueyan, 2018; Zhang et al., 2019). Interpreting pressure transient information from horizontal wells is more involved than for tests in vertical wells, as a consequence of the three dimensional flow geometry impacting horizontal wells that is typically not distributed in a symmetrically radial pattern. Several distinct flow regimes are potentially plausible and, therefore, must be considered when interpreting horizontal pressure transient data (Daviau et al., 1988; Odeh and Babu, 1989; Kuchuk, 1995; Brown et al., 2011; Biryukov and Kuchuk, 2012, 2015; Al-Rbeawi and Tiab, 2013; Torcuk et al., 2013; Kuchuk and Biryukov, 2015; Feng et al., 2017; Duan et al., 2020). Moreover, interpretation models of horizontal wells must consider more extensive wellbore storage effects, the impacts of partial reservoir penetration, and complex end effects.

There are some key differences between well test interpretation applied to horizontal and vertical wells. Whereas, some variables such as average vertical permeability, vertical thickness and skin are used in vertical pressure transient analysis, in horizontal pressure transient analysis some of these variables related to the horizontal geometry, such as vertical and horizontal permeability must be known more specifically. Also, in horizontal wells, there are five potential major and distinguished regimes that can and do commonly occur (Horne, 1995). However, some of these plausible flow regimes may not occur because of wellbore geometrical factors, or are obscured by wellbore storage effects, and other factors. It is therefore possible to estimate reservoir properties from horizontal pressure transient data by applying five different but potentially plausible flow regimes. The five recognized flow regimes in horizontal wells are early radial, hemi-radial, early linear, late pseudo-radial, and late linear (Goode and Thambynayagam, 1987; Lichtenberger, 1994; Luo et al., 2010).

Before the pressure waves pass through the reservoir volume and sense the reservoir boundaries, reservoir fluids produced are only dependent on the vertical boundaries of the reservoir. Following this initial transient flow regime, hemi-radial flow can occur if the horizontal well trajectory approaches much closer to one of the vertical boundaries. Finally, once the portion of the reservoir impacted by fluid production includes the complete reservoir thickness, a linear flow pattern typically develops.

The interpretation of pressure derivative plots is a powerful, and well-stablished tool for analyzing pressure transient data (Al-Rbeawi, 2019). Pressure derivative plots are more useful for extracting a distinctive model compared to the original pressure versus time dataset collected. Such plots are able to specify the type of fluid flow model operating at the reservoirwellbore interface using pattern matching of observed (actual) pressure derivative curves with type curves. To find the appropriate reservoir flow model, the actual field derivative plot is matched to the curve produced by a specific flow regime model, and that enables the correct reservoir properties to be derived (Bourdet et al., 1989).

Chu et al. (2019) applied a convolutional neural network (CNN) to develop a model for identifying different types of well-test curves. They employed some common multi-class classification techniques such as one-hot encoding, Xavier normal initialization, plus some regularization techniques, followed by an Adam optimizer to identify the best model. They concluded that $\mathrm{CNN}$ are more powerful tools for classification of well-test pressure curves in comparison with conventional neural networks of similar structure.

Taibi et al. (2019) proposed a model to detect rock fractures in petroleum reservoirs automatically from well-log data. They developed an algorithm including five different stages and applied them to imaging-log data. Their model commences by extracting the main image features and ultimately locates reservoir fractures using the Hough Transform method. Their developed model performed robustly to detect rock fractures when tested by imaging log data.

Geng et al. (2019) proposed a recognition model approach based on a machine-learning classifier, the AdaBoost algorithm. Their algorithm integrates a model-free Bayesian classifier and the support vector machine algorithm to find a reliable way of detecting water-flooded layers in oil and gas reservoirs. Their classifier model successfully detected the water-flooded layers in the reservoir they evaluated, suggesting that it could be usefully applied in oil and gas reservoir evaluation and aid field development decision making.

An investigation to detect a specified reservoir fluid flow model using artificial neural networks (ANN) was first conducted by Ershaghi et al. (1993). Subsequently, Sung et al. (1996) deployed a multilayer perceptron network involving the Hough transform method to identify various reservoir flow models. Velez-Langs (2005) applied a genetic optimization algorithm for oil reservoir flow regime characterization. Other algorithms such as fuzzy logic, support vector machine, various evolutionary optimization algorithms, and hybrid methods involving several such algorithms, have been applied to detect petroleum reservoir fluid flow systems. Anifowose and Abdulraheem (2011) developed a functional, hybrid network involving a type-2 fuzzy logic system with a support vector machine to model petroleum reservoir fluid flow characterization.

Athichanagorn and Horne (1995) used ANN to identify useful features of pressure derivative curves, including: unit gradients, specific dips or humps, and constant or declining gradients. This approach enabled the appropriate reservoir flow regimes to be identified and matched with reservoir metrics from pressure transient datasets. May and Dagli (1988) established a hybrid neural network model for interpretation which had the benefit of reduced time to train the network. Kharrat and Razavi (2008) applied an ANN model to a dataset of fifty pressure derivative actual curves to successfully identify reservoir flow regimes. The ANN model was trained in each stage to detect different types of reservoir model. To do so involves a classification problem. Hence, the outputANN layer establishes the probability of each trained model matching the input model. Consequently, the number of nodes in the output layer equals the number of trained model types.

Vaferi et al. (2011) used multilayer perceptron (MLP) networks to automatically identify eight distinct reservoir flow regimes from vertical pressure transient data. In order to train and test their ANN model, they employed analytical solutions of commonly occurring reservoir models to generate 
data. Eight reservoir models including heterogenous reservoir conditions such as fractured reservoirs were considered. Various boundary conditions, for instance, constant-pressure outer-boundary no-flow rate, single-sealing-fault boundary and infinite-acting boundary conditions were considered. Ghaffarian et al. (2014) applied MLPs to recognize flow regimes in gas condensate reservoirs, distinguishing twelve distinct reservoir flow models and boundary conditions in vertical wells. They used a radial composite model to reflect gas- condensate reservoir conditions. This required distinguishing two reservoir areas: 1) circular region with the well at its center; and, 2) an infinite outer region. They located an optimal MLP configuration by conducting sensitivity analysis. Each sensitivity case evaluated accuracy criteria by measuring the mean relative error (MRE) and mean squared error (MSE) for various MLP models configured with different hyperparameter values, such as the number of neurons in each hidden layer and the number of hidden layers. Here, we develop a single MLP neural network to recognize and distinguish six different reservoir flow models and boundary conditions in pressure transient data from horizontal wells. Since the pressure response is naturally transient, Vaferi et al. (2015) employed a dynamic ANN model (i.e., recurrent neural network) for observing the dynamic behavior of eight different flow models applied to oil reservoirs with pressure transient data from vertical wellbores. That work considered closed outer boundary reservoir models for both homogenous and dual porosity, which are not considered among the six reservoir models used for this study, although our technique could be also applied to such outer boundary models.

Vaferi et al. (2016) developed a coupling MLP network combined with wavelet transform for detection of reservoir models in vertical pressure transient datasets. The couplingwavelet method reduces the number of pressure signals that are used as inputs to the network, which was not necessary in the current study. Here, we further develop these hybrid-MLP techniques, applying them for the first time to a large well-test dataset specifically focused on horizontal rather than vertical wells.

This work is organized to sequentially:

- Generate synthetic pressure transient signals for the considered reservoir (2560 signals);

- Convert pressure transient signals to the pressure derivative graphs using Eq. (5);

- Normalize of the pressure derivative graphs using Eq. $(6)$;

- Develop different MLP models with different numbers of hidden neurons;

- Compare performance of the developed MLP models to locate the optimal model;

- Apply the most accurate MLP model to a real reservoir example.

\section{Methods}

\subsection{Pressure transient test}

In a pressure drawdown test the flow rate is held ap- proximately constant while the well pressure is continuously measured versus the time elapsed from the beginning of the flow period. This pressure data versus time curve in drawdown test can also be obtained using the solution of the governing equation for the appropriate reservoir fluid flow regime.

Indeed, a range of pressure versus flow period time elapsed curves can be generated from the solution of the governing equation for the appropriate flow regime for a defined reservoir by varying certain reservoir metrics. The pressure response in different parts of the reservoir formation results in movement of fluid towards and into the production well under testing by imposing a constant rate of production. During the early stages of production in the well test, the pressure response changes rapidly due to wellbore storage effects. Subsequently, as time progresses through the test period, the pressure response changes more slowly as it expands further and senses a greater volume of the reservoir formation. Essentially, pressure transient test interpretation is based on the pressure derivative. As time through the test progresses, the pressure derivative value increases, and the pressure response becomes more obvious. This procedure continues until the pressure signal becomes distributed throughout the whole of the formation in communication with the wellbore under test and eventually touches the boundaries of the permeable reservoir formation.

\subsection{Interpretation of reservoir pressure transient responses (PTR)}

Reservoir parameters can be predicted by matching the well test data with an appropriate reservoir fluid flow model. Before reservoir parameters can be estimated with confidence, the appropriate reservoir flow model(s) and boundary conditions need to be selected. Several reservoir flow models and boundary conditions are well known and mathematically defined. Here, we consider homogeneous (single) and dual porosity reservoir flow models (Moosavi et al., 2018a) each with distinct outer and horizontal boundary conditions are considered. These represent the type of oil reservoir geometries into which most horizontal wells are drilled. Infinite acting and constant pressure reservoir flow models are considered as an outer boundary condition, while no-flow/no-flow, noflow/constant pressure boundary conditions constitute the horizontal boundary of the reservoir systems. Although some other reservoir boundary conditions could be evaluated, e.g., such as single fault or closed outer boundary conditions, we elected not to include them but rather focus upon the more commonly applied boundary conditions. These six models are chosen since they are the most common types of reservoir geometry models that are used to evaluate pressure versus time data in horizontal wells.

A summary of these reservoir systems, including the reservoir flow models applied, outer and horizontal boundaries, and numbers of synthetic pressure responses generated for each model are given in Table 1. These datasets (i.e., pressure signals versus time) are generated using an analytical solution for fluid flow partial differential equations using the orthogonal collocation method (Vaferi et al., 2012; Nategh et al. 2019). 
Table 1. Oil reservoir flow models and their specific boundary conditions.

\begin{tabular}{lllll}
\hline System & Reservoir & Horizontal boundary & Outer boundary & $\mathrm{N}^{*}$ \\
\hline Model 1 & Dual porosity & No flow - no flow & Infinite acting & 569 \\
Model 2 & Homogeneous & No flow - no flow & Infinite acting & 532 \\
Model 3 & Homogeneous & No flow - constant pressure & Constant pressure & 473 \\
Model 4 & Homogeneous & No flow - constant pressure & Infinite acting & 255 \\
Model 5 & Dual porosity & no flow - constant pressure & Infinite acting & 160 \\
Model 6 & Homogeneous & No flow - No flow & Constant pressure & 571 \\
\hline
\end{tabular}

* Number of generated synthetic pressure derivative curves.

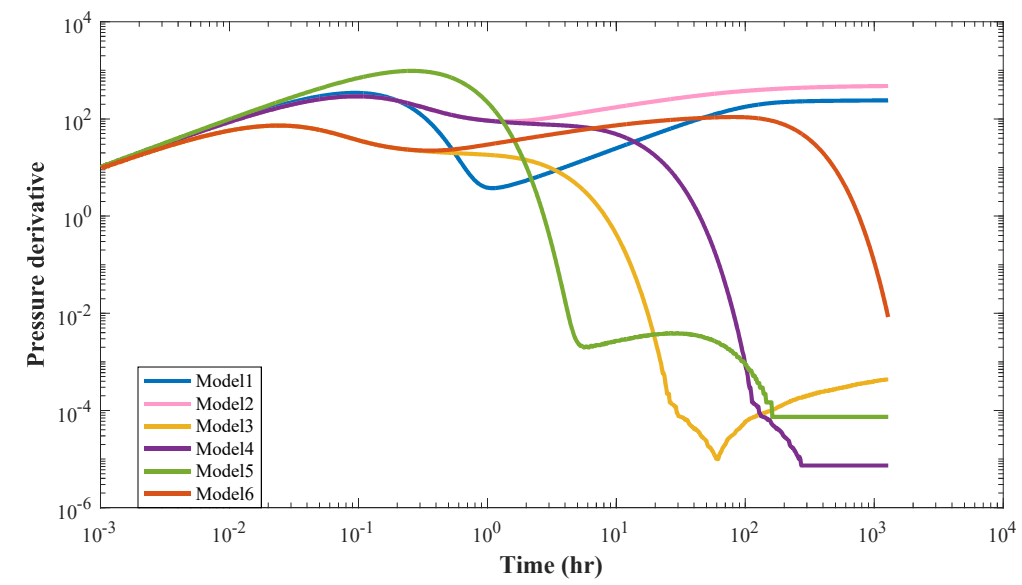

Fig. 1. Typical pressure derivative curves for the considered reservoir models.

The dual porosity reservoir evaluated is a fractured reservoir with two distinct component parts: 1) a fractured section with high fluid transmissibility and low storage capacity; and, 2) the matrix section having low fluid transmissibility and a high storage capacity. Since fluid flow in horizontal wells has three dimensional flow geometry, distinct from a vertical well, the fluid flow model requires two no-flow and noflow/constant pressure boundaries. A horizontal well drilled into a rectangular reservoir with no-flow boundaries at the top and bottom of the reservoir is considered as a two no-flow model. A no-flow/constant pressure boundary model considers a rectangular reservoir with a no-flow boundary at the top or bottom and a constant pressure condition for the other boundary.

The reservoir flow models evaluated in this study assume that the pressure derivative curve is mainly influenced by reservoir geometry factors. The fluid, rock property and wellbore geometry assumptions made in the analysis presented are listed in Table 2. These assumptions imply that several oil composition, rock property, and layer thickness metrics have negligible impacts on pressure derivative curves. Ultimately, such assumptions need to be evaluated and verified, but the objective here is to demonstrate the viability of distinguishing between the most common reservoir flow models using pressure derivative data applying an MLP network technique.

Although the shape of the pressure change curve versus time for all these reservoir systems is similar, their pressure
Table 2. Characteristics of fluid, rock, producing layer, and wellbore.

\begin{tabular}{lll}
\hline Parameter & Value & Unit \\
\hline Well radius & 0.3 & $\mathrm{ft}$ \\
Formation thickness & 50 & $\mathrm{ft}$ \\
Porosity & 0.17 & - \\
Viscosity & 1.2 & $\mathrm{cP}$ \\
Total compressibility & $5 \times 10^{-5}$ & $\mathrm{psi}^{-1}$ \\
Oil formation volume factor & 1.3 & $\mathrm{Rb} / \mathrm{STB}$ \\
Initial pressure & 5000 & $\mathrm{psi}$ \\
Production time & 1300 & $\mathrm{hr}$ \\
Oil flow rate & 650 & $\mathrm{STB} / \mathrm{day}$ \\
\hline
\end{tabular}

derivative curves are quite distinct. Indeed, the MLP approach discriminates among these reservoir systems based on the difference in the characteristic shapes of their pressure derivative curves. Fig. 1 illustrates the characteristic shapes of the pressure derivative curves of the considered reservoir systems described in Table 1.

\subsection{Flow regimes typically encountered in horizontal wells}

Different flow regimes in horizontal wells can occur due to 


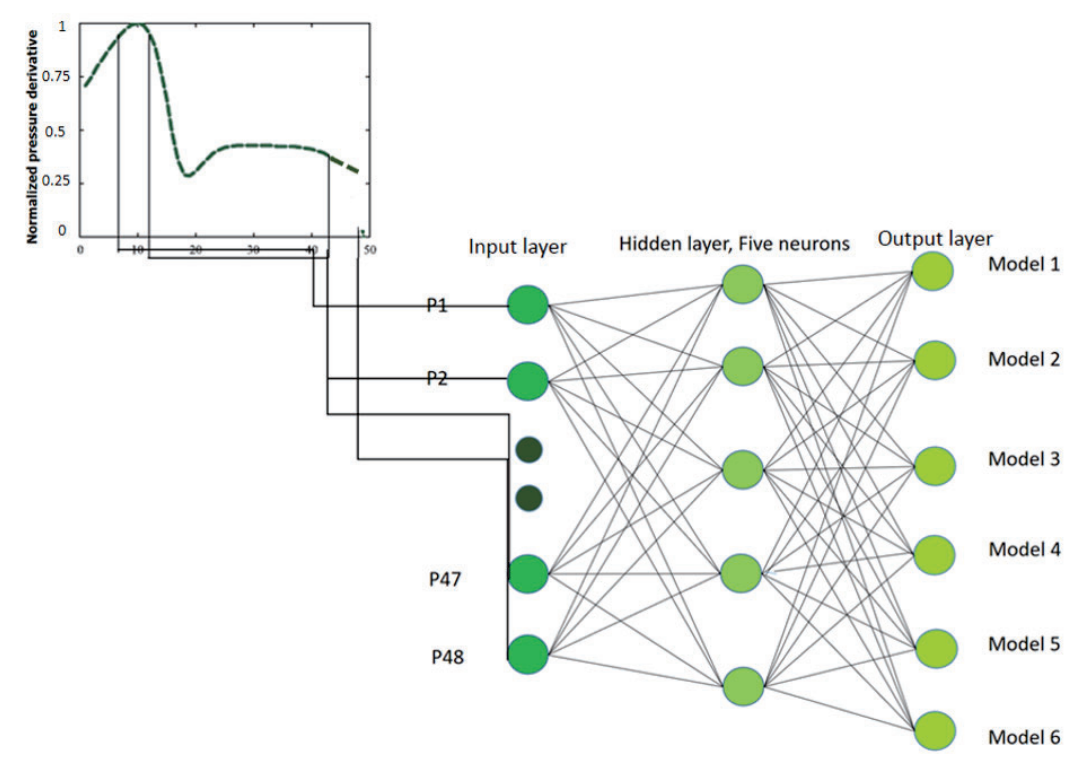

Fig. 2. Schematic of the MLP classifier for reservoir model detection from pressure-derivative curves.

various reservoir geometries. Generally, five main fluid flow regimes are observed (some of them may be absent in some reservoir) in horizontal flowing wells. These include: early radial; hemi-radial; early linear; late pseudo-radial; and, late linear.

Early-radial flow prevails in the early flow period before the pressure transient signal meets any of the vertical boundaries impacting the reservoir. Hemi-radial flow occurs when the well is not located exactly in center of reservoir in the vertical dimension, i.e., the well is closer to one of the vertical boundaries. An early-linear flow pattern may develop when the pressure signal encounters both vertical boundaries. Some fluid flow typically enters a horizontal wellbore from the reservoir zone beyond the end of the drilled section. As such end effects exist in horizontal wells, early-linear flow tends to continue for some time. Eventually, the end effects become negligible, and then a transition period evolves into a later pseudo-radial flow regime. Finally, late-linear flow materializes when the pressure signals expands throughout the entire reservoir and encounters the outer reservoir boundaries (i.e., the infinite acting and constant pressure boundaries assumed by this study) which can be seen in pressure derivative curves (Dikken, 1990; Raghavan et al., 1997).

\subsection{Artificial neural networks}

ANNs are nonlinear, mathematical learning networks based on analogies to human brain neural systems. The networks have several layers with a number of interacting nodes i.e., neurons. These artificial neurons are connected together in a particular way that is specified based on the network architecture. The output neurons can be calculated using Eq. (1).

$$
\text { net }=\sum(W \times X)+b
$$

where $W$ is the weight matrix between neurons in each layer, $b$ refers to the bias vector, and, net represents entry signal to the transfer function $(f)$.

Indeed, the results of Eq. (1) are entered into the transfer function to calculate the value of neuron output (out). This can be simply expressed as Eq. (2).

$$
\text { out }=f\left(\sum(W \times X)+b\right)
$$

There are various types of transfer functions that are commonly applied to ANN. Examples of the activation or transfer functions applied include tangent sigmoid, logarithm sigmoid, linear and radial basis functions are. In this work, we use tangent sigmoid, Eq. (3), and logarithm sigmoid, Eq. (4), as the activation function between the input and hidden layers and hidden and output layers, respectively.

$$
\begin{gathered}
f(\text { net })=\frac{e^{n e t}-e^{-n e t}}{e^{n e t}+e^{-n e t}} \\
f(n e t)=\frac{1}{1+e^{-n e t}}
\end{gathered}
$$

where $f($ net) is the output of the neuron, which becomes the input for the neuron it is connected to in the next layer or provides the network output. Fig. 2 illustrates the architecture of the ANN model developed highlighting the input of data from forty eight segments of the pressure derivative curves into the network.

Each of the 48 input samples from the pressure derivative curve is taken at a specific time elapsed point during the synthetic well test operation. The time elapsed points are the same for each input curve, making the time intervals for the derivatives fixed and avoiding the need to consider time as a dependent variable for model detection.

Similar simulation time, and time elapsed intervals are used to generate the synthetic pressure transient data for each case. Each pressure versus time curve generated has 50 data points. During transformation of the pressure data into pressure derivative data (applying Eq. (5)), the first and the last pressure 
data-point in the pressure curve are diminished (they contribute to the first and last derivative with their adjacent data points). Therefore 48 pressure derivative data-points are calculated and fed into the MLP network (see Fig. 2) for detection of reservoir models. Since the absolute times and the time intervals of each derivative point are the same for each pressure derivative curve generated, time has no influences on the network's ability to detect the underlying reservoir flow model determining each pressure derivative curve. Therefore, it is only the 48 pressure derivative data values for each curve evaluated that need to be input to the MLP network; absolute time elapsed or time interval values are not required.

It is clear that the MLP neural network approach recognizes the actual reservoir model based on differences in the shape of the pressure derivative curves considered. Therefore, by increasing the number of data points for each pressure derivative curve, it is possible to improve the resolution of the curves of each reservoir flow model introduced to the MLP network. This helps the network achieve better pattern recognition. On the other hand, by increasing the numbers of data points constituting each pressure derivative curve, the size of the MLP neural network (i.e., the number of weights and biases), computational effort and its complexity sharply increases. By performing trial and error sensitivities on the number of data points associated with each pressure derivative curve, we conclude that 50 pressure versus time data points (resulting in 48 pressure derivative data points per curve) is the optimum value effectively trading-off curve resolution and computational complexity.

\subsubsection{Pre-processing requirements for synthetic pressure transient responses}

A large number of pressure transient signals for drawdown tests for the considered reservoir flow systems are initially simulated by solving their diffusivity equations. A range of values for key properties relating to the wellbore, the reservoir, and the boundaries (Table 3) are selected as inputs to the simulation to generate synthetic pressure responses for each of the considered reservoir flow systems.

Bottom-hole pressure transient curves are not unique in shape and form for each reservoir flow and boundary system studied. As pressure derivative curves are regarded as one of the best reservoir flow model discrimination tools, in this study, it is the pressure derivative curves that are employed to distinguish between the reservoir flow systems under consideration. The method of Bourdet is used to transform the synthetic pressure transient data into pressure derivative curves. Bourdet's algorithm is expressed as Eq. (5).

$$
d P=\frac{\left\{\begin{array}{c}
\frac{(p)_{k}-(p)_{k-1}}{\ln (t)_{k}-\ln (t)_{k-1}}\left[\ln (t)_{k+1}-\ln (t)_{k}\right] \\
+\frac{(p)_{k+1}-(p)_{k}}{\ln (t)_{k+1}-\ln (t)_{k}}\left[\ln (t)_{k}-\ln (t)_{k-1}\right]
\end{array}\right\}}{\ln (t)_{k+1}-\ln (t)_{k-1}}
$$

where $d P$ is the pressure derivative, $p$ is pressure drop, $t$ is time, and, $k$ is the sample number in the time sequence.

Pressure derivative data (to be used for both MLP training and testing subsets) are then normalized to values within the interval [0 1] using Eq. (6). This helps the network to convergence more easily, prevents saturation of its parameters and biases due to metrics with values of significantly different value ranges.

$$
\overline{d P}=\frac{d P-d P_{\min }}{d P_{\max }-d P_{\min }}
$$

\subsubsection{Training stage of the MLP classifier}

The ANN model developed here to recognize and distinguish reservoir flow systems is an MLP network with a feedforward structure. Its evaluation algorithm evaluates 2560 synthetic pressure derivative curves. $90 \%$ of the full dataset

Table 3. Ranges of key parameters used to generate pressure transient signals

\begin{tabular}{lllll}
\hline Reservoir model & Parameters & Unit & Minimum & Maximum \\
\hline & $K$ & $\mathrm{md}$ & 2 & 180 \\
Homogenous reservoir & $S$ & - & -1 & 2 \\
& $W_{s c}$ & $\mathrm{bbl} / \mathrm{psi}$ & 0.001 & 0.1 \\
& $K_{v}$ & $\mathrm{md}$ & 2 & 50 \\
& $L_{w}$ & $\mathrm{ft}$ & 400 & 1200 \\
\hline \multirow{3}{*}{ Dual porosity reservoir } & $K$ & $\mathrm{md}$ & 10 & 320 \\
& $S$ & - & -1 & 2 \\
& $W_{s c}$ & $\mathrm{bbl} / \mathrm{psi}$ & 0.001 & 0.01 \\
& $\omega$ & - & 0.001 & 0.1 \\
& $\lambda$ & - & $1 \times 10^{-8}$ & $1 \times 10^{-5}$ \\
& $K_{v}$ & $\mathrm{md}$ & 2 & 50 \\
& $L_{w}$ & $\mathrm{ft}$ & 400 & 1200 \\
\hline
\end{tabular}

Note: $K=$ permeability, $S=$ Skin factor, $W_{s c}=$ wellbore storage coefficient, $K_{v}=$ vertical permeability, $L_{w}=$ well length, $\omega=$ Storativity ratio, $\lambda=$ Interporosity flow coefficient. 
Table 4. Sensitivity analyses results used to select the optimum MLP network structure.

\begin{tabular}{|c|c|c|c|c|}
\hline Number of hidden neurons & $T C A(\%)$ & Database & $M S E$ & $R^{2}$ \\
\hline & & Training & 0.65989 & 0.51832 \\
\hline \multirow[t]{3}{*}{1} & 70.5 & Testing & 0.69145 & 0.48718 \\
\hline & & Overall & 0.66305 & 0.51517 \\
\hline & & Training & 0.29417 & 0.79682 \\
\hline \multirow[t]{3}{*}{2} & 82.4 & Testing & 0.30241 & 0.79066 \\
\hline & & Overall & 0.29499 & 0.79619 \\
\hline & & Training & 0.15347 & 0.89904 \\
\hline \multirow[t]{3}{*}{3} & 90.8 & Testing & 0.18418 & 0.87755 \\
\hline & & Overall & 0.15653 & 0.89690 \\
\hline & & Training & 0.09419 & 0.93953 \\
\hline \multirow[t]{3}{*}{4} & 96.1 & Testing & 0.10153 & 0.93458 \\
\hline & & Overall & 0.09492 & 0.93904 \\
\hline & & Training & 0.00721 & 0.97330 \\
\hline \multirow[t]{3}{*}{5} & 98.3 & Testing & 0.00737 & 0.97349 \\
\hline & & Overall & 0.00725 & 0.97332 \\
\hline & & Training & 0.06039 & 0.96183 \\
\hline \multirow[t]{3}{*}{6} & 97.2 & Testing & 0.11762 & 0.92462 \\
\hline & & Overall & 0.06610 & 0.95811 \\
\hline & & Training & 0.08232 & 0.94727 \\
\hline \multirow[t]{3}{*}{7} & 95.4 & Testing & 0.10180 & 0.93442 \\
\hline & & Overall & 0.08426 & 0.94599 \\
\hline & & Training & 0.06782 & 0.95709 \\
\hline \multirow[t]{3}{*}{8} & 98.0 & Testing & 0.07056 & 0.95538 \\
\hline & & Overall & 0.06809 & 0.95692 \\
\hline & & Training & 0.06798 & 0.95687 \\
\hline \multirow[t]{2}{*}{9} & 97.3 & Testing & 0.06060 & 0.96158 \\
\hline & & Overall & 0.06724 & 0.95734 \\
\hline
\end{tabular}

of pressure derivative curves are utilized for training the MLP, and the remaining $10 \%$ are used for independently testing the performance of the trained MLP neural network.

As the dataset includes six distinct reservoir flow models, the MLP needs to distinguish those, and to do so the number of outputs neurons for the MLP set to six. During network training, each output neurons takes an integer value of 0 or 1 to indicate the possibility of it owning each input pressure derivative curve, i.e., assigning each input curve to a particular reservoir flow system. The topology of the MLP is assessed by trial and error to establish the appropriate number of neurons in its single hidden layer. Cybenko (1989) found out that MLPs with just a single hidden layer can predict most types of nonlinear systems. Therefore, the MLP design employed here for detecting reservoir flow models involves a single hidden layer, which the results obtained suggest is appropriate. Computational time required for training the MLP is one of the factors considered in selecting the best training algorithm. Additionally, three statistical prediction performance measures are used to select the optimum number of neurons in the hidden layer of the MLP network. These are: the mean squared error, the regression coefficient, and the total classification accuracy (TCA) defined by Eq. (7):

$$
T C A=\frac{\left(\begin{array}{c}
\text { Total number of correct detections } \\
\text { of reservoir flow model }
\end{array}\right)}{\left(\begin{array}{c}
\text { Total number of pressure } \\
\text { derivative curves tested }
\end{array}\right)} \times 100
$$

where TCA determines the fraction of all the synthetic pressure derivative curves for all the reservoir systems studied that are identified accurately. The statistical accuracy of the MLP network is affected by the initial random values of bias and weight coefficients. Consequently, exact values for $W$ and $b$ for correct detection of reservoir model are evaluated during the training stage of the MLP network.

\section{Results and discussion}

\subsection{Selecting the optimum configuration for the MLP classifier}

The statistical measures of accuracy for different MLP 
Table 5. Performance comparison of applying ten different training algorithms to the developed MLP neural network with 5 hidden neurons.

\begin{tabular}{lllll}
\hline Training algorithm & $T C A(\%)$ & $M S E$ & $R^{2}$ & Training speed $^{a}$ \\
\hline Conjugate gradient back propagation with PowellBeale restarts & 83.3 & 0.22273 & 0.85110 & 0.0128 \\
Conjugate gradient back propagation with PolakRibiere updates & 85.8 & 0.18923 & 0.87502 & 0.0088 \\
Gradient descent with momentum back propagation & 23 & 0.86446 & 0.24815 & 0.0035 \\
Gradient descent with adaptive learning rate & 51.8 & 0.38413 & 0.72364 & 0.003 \\
Scaled conjugate gradient back propagation & $\mathbf{9 8 . 3}$ & $\mathbf{0 . 0 0 7 2 5}$ & $\mathbf{0 . 9 7 3 3 2}$ & $\mathbf{0 . 0 0 4 2}$ \\
Gradient descent with momentum and adaptive learning rate back & 45.5 & 0.36050 & 0.74274 & 0.0028 \\
propagation & 100 & 0.00280 & 0.99831 & 0.3886 \\
Levenberg-Marquardt back propagation & 89.8 & 0.11864 & 0.92494 & 0.0967 \\
BFGS quasi-Newton back propagation & 99.4 & 0.00695 & 0.99578 & 0.3669 \\
Bayesian regulation back propagation & 68.3 & 0.25164 & 0.82970 & 0.0104 \\
One-step secant back propagation & & & \\
\hline
\end{tabular}

${ }^{a}$ In second/epoch by installed memory $(\mathrm{RAM})=4.00 \mathrm{~GB}$, and $3.3 \mathrm{GHz}$ Intel (R) Pentium (R) CPU

Table 6. The confusion matrix for the MLP classifier over whole datasets.

\begin{tabular}{lllllll}
\hline Desired/Output & Model 1 & Model 2 & Model 3 & Model 4 & Model 5 & Model 6 \\
\hline Model 1 & 569 & 0 & 0 & 0 & 0 & 0 \\
Model 2 & 0 & 532 & 0 & 0 & 0 & 0 \\
Model 3 & 0 & 0 & 467 & 6 & 0 & 0 \\
Model 4 & 0 & 0 & 8 & 0 & 0 & 1 \\
Model 5 & 0 & 2 & 0 & 0 & 0 & 0 \\
Model 6 & 0 & 0 & 0 & 0 & 0 & 571 \\
\hline
\end{tabular}

network structures with various numbers of neurons in the hidden layer are reported in Table 4 . The configuration with 5 neurons has the smallest MSE and the highest TCA and $R^{2}$ value, i.e., the network with the best topology.

The MLP with just five neurons in the hidden layer was able to detect correctly to which reservoir flow system belonged 2516 (from 2560) of the pressure derivative curves for horizontal wells evaluated. It achieved this with $T C A=$ $98.3 \%, R^{2}=0.97332$, and $M S E=0.00725$.

\subsection{Selection of the best training algorithm}

During the training stage the weights and biases of the optimum MLP network are selected that achieve high prediction accuracy with the minimum of computational efforts. This was established by considering the taken time by the computer central processing unit (CPU), together with the defined statistical measures of prediction accuracy, to select the most efficient MLP training algorithm. The average CPU time and the statistical accuracy measures (i.e., TCA, MSE, and $R^{2}$ ) achieved by ten of the most widely used MLP training algorithms are compared in Table 5.

Although the Levenberg-Marquardt back propagation achieved perfect prediction accuracy for the dataset evaluated, the method of scaled conjugate gradient back propagation is chosen as the most efficient MLP training algorithm from the results presented in Table 5. This is because it is able to provide acceptable accuracy with very small computational effort. Although the obtained TCA by the scaled conjugate gradient is $1.7 \%$ lower than the $T C A$ of the LevenbergMarquardt, it compensates for that small error by requiring much less computational effort. The CPU time required by the Levenberg-Marquardt algorithm is about 93 times higher than the CPU time required by the scaled conjugate gradient algorithm. Training starts with random values selected for the weights and biases and therefore the first stage has the highest errors. By adjusting these parameters, the training algorithm manages to progressively reduce MSE to very low values, indicative of high prediction accuracy. The scaled conjugate gradient algorithm has adjusted the $W$ and $b$ parameter of the MLP network after 3000 iterations to reduce the MSE to $7.21 \times 10^{-3}$ for the training data subset.

\subsection{Accuracy of the MLP classifier for reservoir detection from smooth PTRs}

The recognition response of trained MLP network for test data is provided in Table 6 , by identifying the number of correct diagnosis for each pressure derivative curve tested. The trained MLP network is clearly able to recognize pressure derivative curves and allocate them accurately to the appropriate reservoir flow models for all the reservoir geometries considered. For a specified pressure derivative curve as an input, the MLP network could select more than one reservoir 
Table 7. Classification accuracy for the developed MLP over whole databank.

\begin{tabular}{lllllll}
\hline Performance (\%) & Model 1 & Model 2 & Model 3 & Model 4 & Model 5 & Model 6 \\
\hline Sensitivity & 100 & 100 & 98.7 & 94.5 & 96.1 & 100 \\
$T C A$ & & & & 98.3 & & \\
\hline
\end{tabular}

Table 8. Used parameters for investigation of extrapolation ability of the MLP classifier.

\begin{tabular}{llllllll}
\hline Reservoir system & $K$ & $S$ & $W_{s c}$ & $K_{v}$ & $L_{w}$ & $\omega$ & $\lambda$ \\
\hline Model 1 & 380 & -2 & 0.0008 & 60 & 1300 & 0.0001 & $1 \times 10^{-9}$ \\
Model 2 & 400 & 2.3 & 0.0005 & 58 & 350 & - & - \\
Model 3 & 75 & -1.4 & 0.0007 & 1.6 & 1340 & - & - \\
Model 4 & 360 & -1.8 & 0.0009 & 62 & 380 & - & \\
Model 5 & 355 & 1400 & 0.00085 & 53 & 390 & 0.0005 & $-1 \times 10^{-4.5}$ \\
Model 6 & 60 & 400 & 0.0006 & 1.8 & 1400 & - & - \\
\hline
\end{tabular}

flow model, because some pressure versus time data patterns can be similar and not easily allocated to a single specific reservoir flow model.

The number of synthetic pressure-derivative curves generated for reservoir flow model 4 and model 5 are 255 and 160, respectively (See Table 1). Table 5 reveals that for model 4 and model 5 the outputs of $(241+8+1=250)$ and $(154+2=$ 156) patterns are displayed, respectively. This occurs because we set the objective selection probability to be above $60 \%$. Hence, Table 6 does not include the pressure derivative curves which have an assignment accuracy below $60 \%$ (i.e., 5 curves for reservoir flow model $4 ; 4$ curves for reservoir flow model $5)$.

Network detection sensitivity and TCA are two statistical indices which are used here to quantify the identification efficiency of the developed MLP network. Table 7 reports ratios the number of correctly identified reservoir flow models relating to a particular reservoir flow model to the number of all the pressure derivative curves tested for that model. The sensitivity index is defined by Eq. (8).

$\operatorname{Sensitivity}(\%)=\frac{\left(\begin{array}{c}\text { Total correct detections of } \\ \text { given reservoir-flow system }\end{array}\right)}{\left(\begin{array}{c}\text { Total derivative-pressure curves } \\ \text { for a given reservoir-flow system }\end{array}\right)} \times 100$

\subsection{The MLP classifier for reservoir model detection from extrapolated signals}

An additional range of reservoir system metric values are employed for generating synthetic pressure time data for each specified reservoir model (Table 8). This additional data (six data sets for each of the six reservoir flow models) is used to evaluate the ability of the MLP network to identify reservoir system types beyond the training and testing data.

Table 9 presents the output of the trained MLP network

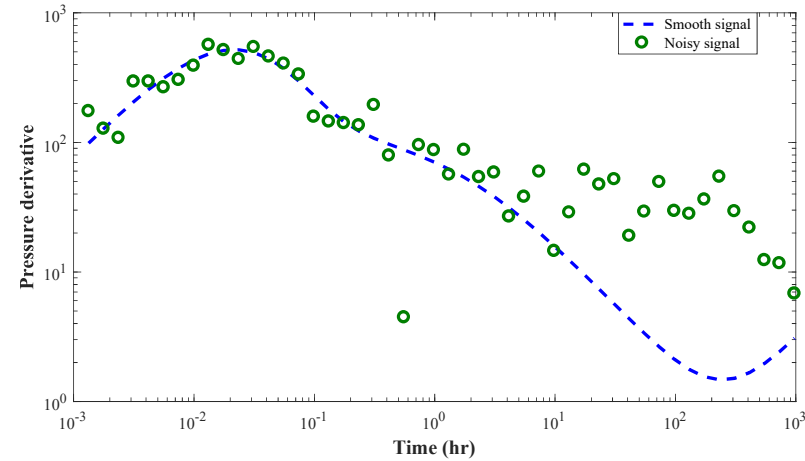

Fig. 3. Pressure derivative curve for a noisy and a smooth pressure signal.

response to additional synthetic pressure transient data (six data sets/reservoir flow model). These results indicate that the correct reservoir flow models for each of the six models evaluated can be successfully recognized using the trained MLP network with acceptable levels of accuracy. The MLP network output values listed in Table 9 represent the probabilities of specific pressure derivative curves being assigned to one or other of the six reservoir flow models. The trained MLP network recognizes the additionally generated pressure derivative curves and assigns them correctly to the appropriate reservoir flow model. It does so with probabilities of $88 \%$ or higher for each of the six reservoir flow models studied.

\subsection{Performance of the MLP classifier for reservoir detection from noisy PTRs}

Since real field pressure transient data records are often noisy (unlike the synthetic pressure transient data which is smoothed), the ability and performance of the developed MLP network to detect and correctly assign noisy pressure transient data needs to be established. For this purpose, artificial normal noise was added to one of the data sets of each of the six reservoir flow models evaluated (Moosavi et al., 2018b). Gaussian noise with zero mean and unit variance is added to 
Table 9. Performance of the MLP classifier for detection of reservoir model from extrapolated pressure transient signals.

\begin{tabular}{lllllll}
\hline Predict/Actual & Model 1 & Model 2 & Model 3 & Model 4 & Model 5 & Model 6 \\
\hline Model 1 & $\mathbf{9 9 . 3 2}$ & 0.38 & 0.00 & 0.18 & 0.15 & 0.01 \\
Model 2 & 0.10 & $\mathbf{9 9 . 2 3}$ & 0.03 & 0.17 & 0.35 & 0.01 \\
Model 3 & 0.01 & 0.00 & $\mathbf{9 9 . 8 3}$ & 0.00 & 0.14 & 0.02 \\
Model 4 & 0.00 & 0.00 & 8.62 & $\mathbf{8 8 . 8 6}$ & 2.53 & 0.00 \\
Model 5 & 7.38 & 0.00 & 0.10 & 1.07 & $\mathbf{9 2 . 9 8}$ & 0.00 \\
Model 6 & 0.14 & 0.60 & 0.00 & 0.00 & 0.00 & $\mathbf{9 9 . 2 6}$ \\
\hline
\end{tabular}

Table 10. Response of the MLP classifier to the noisy signals of all six reservoir models.

\begin{tabular}{lllllll}
\hline Input/Output & Model 1 & Model 2 & Model 3 & Model 4 & Model 5 & Model 6 \\
\hline Model 1 & $\mathbf{9 2 . 6 8}$ & 2.21 & 0.03 & 5.03 & 0.01 & 0.04 \\
Model 2 & 1.45 & $\mathbf{9 3 . 1 0}$ & 2.03 & 0.43 & 5.35 & 0.02 \\
Model 3 & 2.09 & 1.32 & $\mathbf{8 9 . 1 3}$ & 0.00 & 3.14 & 6.42 \\
Model 4 & 0.87 & 4.30 & 7.24 & $\mathbf{8 1 . 8 6}$ & 2.53 & 11.72 \\
Model 5 & 9.20 & 1.94 & 3.50 & 1.25 & $\mathbf{8 7 . 6 4}$ & 1.98 \\
Model 6 & 6.41 & 1.82 & 0.87 & 0.00 & 3.34 & $\mathbf{9 1 . 9 6}$ \\
\hline
\end{tabular}

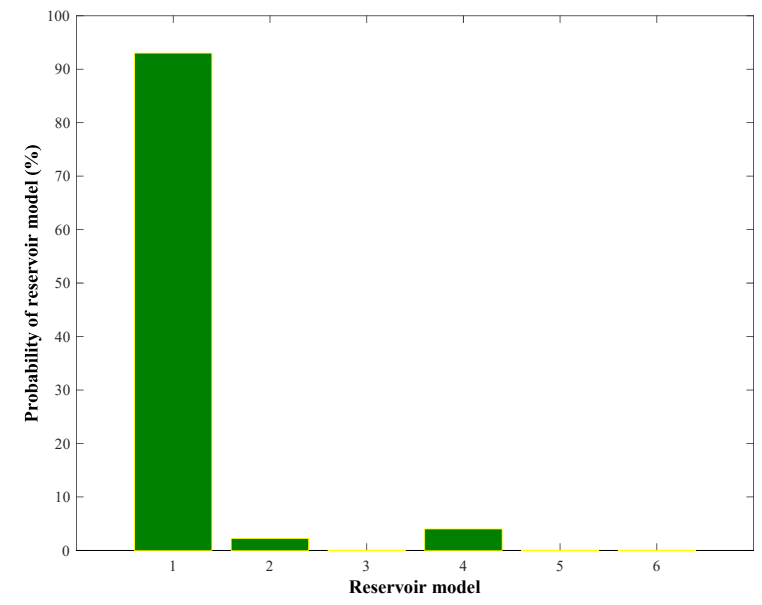

Fig. 4. Response of the MLP classifier to the smooth pressure derivative curve of reservoir model 1 .

the synthetic data sets. To achieve noise at a realistic level, we added random noise in small fractions of $0.5 \%$ to the original pressure signal.

Fig. 3 illustrates a smooth and a noisy pressure derivative curve for model 1 ( $2 \%$ noise). The probability of each of the six reservoir flow models being selected for smooth and noisy data for a reservoir flow model 1 pressure derivative curve is also shown in Figs. 4 and 5, respectively. The results suggest that data noise within the pressure transient data does not affect the prediction accuracy of the trained MLP network, at least in the case of those pressure derivative curves belonging to reservoir flow model 1.

The results of detection performance for noisy pressure derivative signals are presented for each reservoir flow model studied in Table 10. The results show that the detection performance of the MLP network is not affected by data noise

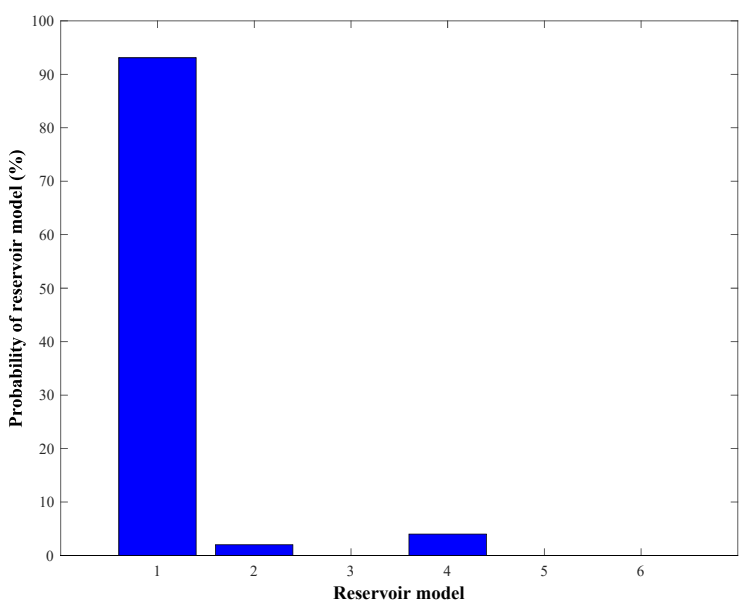

Fig. 5. Output of the MLP classifier to the noisy pressure derivative curve of reservoir model 1.

and the true reservoir flow model is identified in each case with probabilities of between $81 \%$ and $93 \%$.

\subsection{Capability of the MLP classifier in real field conditions}

The efficiency of the developed MLP network in detecting the correct reservoir model is examined for horizontal pressure transient data derived from a real oil field. The real field data selected for this evaluation is that reported by Suzuki and Nanba (1990) for a drawdown test in a single porosity limestone oil reservoir with two no-flow boundaries drilled by a horizontal well. The reservoir fluid is oil during production time since the observed minimum bottom-hole pressure observed is far above the bubble point pressure and negligible production of formation water is observed. 


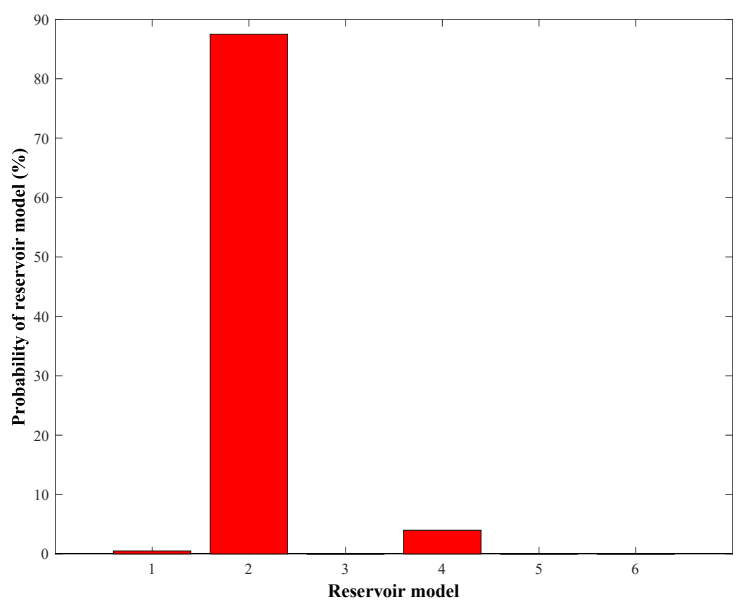

Fig. 6. Response of the classifier to the real field pressure derivative curve (Suzuki and Nanba, 1990).

The normalized data of this case study is then analyzed using the trained MLP. Fig. 6 illustrates that the trained MLP network recognizes and selects the appropriate reservoir flow model with a probability of $88 \%$.

An alternative way to model pressure transient analysis in horizontal wells in fractured reservoirs is to consider pressure build-up analysis rather than pressure drawdown analysis. Future research is planned to adapt the proposed models to focus on pressure build-up data.

\section{Conclusions}

- An automated machine-learning model based on a MLP network for classification of the governing flow models in horizontal wells is developed and successfully applied to synthetic, noisy, and real field pressure-transient signals.

- This smart model is designed to discriminate among homogeneous and dual porosity reservoirs with different outer and horizontal boundary conditions (constant pressure, infinite acting, and no flow).

- Optimum architecture of the MLP network and an appropriate training algorithm are selected based upon classification accuracy and computational time, respectively.

- Based on these criteria, an MLP network was selected with one hidden layer containing five neurons trained using a scaled conjugate gradient feedforward algorithm which was identified as the most efficient classifier.

- This proposed MLP configuration correctly detected 2560 synthetic pressure derivative curves with a total classification accuracy of $98.3 \%$.

- The scaled conjugate gradient back propagation algorithm only required 0.0042 second per epoch for training the MLP classifier.

- The reliability of predictions achieved using the trained MLP network is impressive when applied to noisy pressure transient data sets and well test data from real reservoirs of known reservoir flow conditions drilled by horizontal wells.

- In both cases the trained MLP network identified the correct reservoir flow model with almost a $90 \%$ probability.

\section{Acknowledgement}

The authors wish to thank colleagues at their respective institutions for advice while conducting this study.

\section{Conflict of interest}

The authors declare no competing interest.

Open Access This article, published at Yandy Scientific Press on behalf of the Division of Porous Flow, Hubei Province Society of Rock Mechanics and Engineering, is distributed under the terms and conditions of the Creative Commons Attribution (CC BY-NC-ND) license, which permits unrestricted use, distribution, and reproduction in any medium, provided the original work is properly cited.

\section{References}

Al-Rbeawi, S. Restoring disrupted data by wellbore storage effect using analytical models and type-curve matching techniques. Int. J. Oil Gas Coal Technol. 2018, 19(2): 163-196.

Al-Rbeawi, S., Tiab, D. Pressure behaviours and flow regimes of a horizontal well with multiple inclined hydraulic fractures. Int. J. Oil Gas Coal Technol. 2013, 6(1-2): 207-241.

Anifowose, F., Abdulraheem, A. Fuzzy logic-driven and SVMdriven hybrid computational intelligence models applied to oil and gas reservoir characterization. J. Nat. Gas Sci. Eng. 2011, 3(3): 505-517.

Athichanagorn, S., Horne, R.N. Automatic parameter estimation from well test data using artificial neural network. Paper SPE 30566 Presented at Annual Technical Conference and Exhibition, Dallas, Texas, USA, 22-25 October, 1995.

Biryukov, D., Kuchuk, F.J. Transient pressure behavior of reservoirs with discrete conductive faults and fractures. Transp. Porous Media 2012, 95(1): 239-268.

Biryukov, D., Kuchuk, F.J. Pressure transient behavior of horizontal wells intersecting multiple hydraulic fractures in naturally fractured reservoirs. Transp. Porous Media 2015, 110(3): 369-408.

Bourdet, D., Ayoub, J.A., Pirard, Y.M. Use of pressure derivative in well test interpretation. SPE Form. Eval. 1989, 4(2): 293-302.

Brown, M., Ozkan, E., Raghavan, R., et al. Practical solutions for pressure-transient responses of fractured horizontal wells in unconventional shale reservoirs. SPE Reserv. Eval. Eng. 2011, 14(6): 663-676.

Chu, H., Liao, X., Dong, P., et al. An automatic classification method of well testing plot based on convolutional neural network (CNN). Energies 2019, 12(15): 2846.

Cybenko, G.V. Approximation by superposition of a sigmoidal function. Math. Control Signal. Syst. 1989, 2(4): 303-314.

Daviau, F., Mouronval, G., Bourdarot, G., et al. Pressure analysis for horizontal wells. SPE Form. Eval. 1988, 3(4): 716-724.

Dikken, B.J. Pressure drop in horizontal wells and its effect on production performance. J. Pet. Technol. 1990, 42(11): 1426-1433.

Duan, Y., Ren, K., Fang, Q., et al. Pressure transient 
analysis for a horizontal well in heterogeneous carbonate reservoirs using a linear composite model. Math. Probl. Eng. 2020, 2020: 3267458.

Ershaghi, I., Li, X., Hassibi, M. A robust neural network model for pattern recognition of pressure transient test data. Paper SPE 26427 Presented at SPE Annual Technical Conference and Exhibition, Houston, Texas, USA, 3-6 October, 1993.

Feng, Q., Xia, T., Wang, S., et al. Pressure transient behavior of horizontal well with time-dependent fracture conductivity in tight oil reservoirs. Geofluids 2017, 2017: 5279792.

Geng, Z., Hu, X., Ding, N., et al. A pattern recognition modeling approach based on the intelligent ensemble classifier: Application to identification and appraisal of water-flooded layers. Proc. Inst. Mech. Eng. Part I-J Syst. Control Eng. 2019, 233(7): 737-750.

Ghaffarian, N., Eslamloueyan, R., Vaferi, B. Model identification for gas condensate reservoirs by using ANN method based on well test data. J. Pet. Sci. Eng. 2014, 123: 20-29.

Goode, P.A., Thambynayagam, R.K.M. Pressure drawdown and buildup analysis of horizontal wells in anisotropic media. SPE Form. Eval. 1987, 2(4): 683-697.

Horne, R.N. Modern Well Test Analysis. Palo Alto, USA, Petroway Inc., 1995.

Kharrat, R., Razavi, S.M. Determination of reservoir model from well test data, using artificial neural network. Sci. Iran. 2008, 15(4): 487-493.

Kuchuk, F., Biryukov, D. Pressure-transient tests and flow regimes in fractured reservoirs. SPE Reserv. Eval. Eng. 2015, 18(2): 187-204.

Kuchuk, F.J. Well testing and interpretation for horizontal wells. J. Pet. Technol. 1995, 47(1): 36-41.

Lichtenberger, G.J. Data acquisition and interpretation of horizontal well pressure transient tests. J. Pet. Technol. 1994, 46(2): 157-162.

Luo, S., Neal, L., Arulampalam, P., et al. Flow regime analysis of multi-stage hydraulically-fractured horizontal wells with reciprocal rate derivative function: Bakken case study. Paper SPE 137514 Presented at Canadian Unconventional Resources and International Petroleum Conference, Calgary, Alberta, Canada, 19-21 October, 2010.

May, E.A., Dagli, C.H. A hybrid system for well test analysis. Paper Presented at 2nd IEEE World Congress on Computational Intelligence (WCCI 98), Anchorage, Alaska, USA, 4-9 May, 1988.

Meng, M., Chen, Z., Liao, X., et al. A well-testing method for parameter evaluation of multiple fractured horizontal wells with non-uniform fractures in shale oil reservoirs. Adv. Geo-Energy Res. 2020, 4(2): 187-198.

Moosavi, S.R., Qajar, J., Riazi, M. A comparison of methods for denoising of well test pressure data. J. Pet. Explor. Prod. Technol. 2018b, 8(4): 1519-1534.

Moosavi, S.R., Vaferi, B., Wood, D.A. Applying orthogonal collocation for rapid and reliable solutions of transient flow in naturally fractured reservoirs. J. Pet. Sci. Eng. 2018a, 162: 166-179.

Nategh, M., Vaferi, B., Riazi, M. Orthogonal collocation method for solving the diffusivity equation: application on dual porosity reservoirs with constant pressure outer boundary. J. Energy Resour. Technol. 2019, 141(4): 042001.

Odeh, A.S., Babu, D.K. Transient flow behavior of horizontal wells, pressure drawdown, and buildup analysis. Paper SPE 18802 Presented at SPE California Regional Meeting, Bakersfield, California, USA, 5-7 April, 1989.

Raghavan, R.S., Chen, C., Agarwal, B. An analysis of horizontal wells intercepted by multiple fractures. SPE J. 1997, 2(3): 235-245.

Sung, W., Yoo, I., Ra, S., et al. Development of HT-BP neural network system for the identification of well test Interpretation model. Paper SPE 30974 Presented at SPE Eastern Regional Meeting, Morgantown, West Virginia, USA, 18-20 September, 1995.

Suzuki, K., Nanba, T. Horizontal well test analysis system. Paper SPE 20613 Presented at SPE Annual Technical Conference and Exhibition, New Orleans, Louisiana, USA, 23-26 September, 1990.

Taibi, F., Akbarizadeh, G., Farshidi, E. Robust reservoir rock fracture recognition based on a new sparse feature learning and data training method. Multidimens. Syst. Signal Process. 2019, 30(4): 2113-2146.

Torcuk, M.A., Kurtoglu, B., Fakcharoenphol, P., et al. Theory and application of pressure and rate transient analysis in unconventional reservoirs. Paper SPE 166147 Presented at SPE Annual Technical Conference and Exhibition, New Orleans, Louisiana, USA, 30 September-2 October, 2013.

Vaferi, B., Eslamloueyan, R. Characterisation of hydrocarbon reservoirs by analysing deconvolved impulse response. Int. J. Oil. Gas Coal Technol. 2018, 17(2): 129-142.

Vaferi, B., Eslamloueyan, R., Ayatollahi, S. Automatic recognition of oil reservoir models from well testing data by using multilayer perceptron networks. J. Pet. Sci. Eng. 2011, 77(3-4): 254-262.

Vaferi, B., Eslamloueyan, R., Ayatollahi, S. Application of recurrent networks to classification of oil reservoir models in well-testing analysis. Energy Sources Part A-Recovery Util. Environ. Eff. 2015, 37(2): 174-180.

Vaferi, B., Eslamloueyan, R., Ghaffarian, N. Hydrocarbon reservoir model detection from pressure transient data using coupled artificial neural network-Wavelet transform approach. Appl. Soft. Comput. 2016, 47: 63-75.

Vaferi, B., Salimi, V., Dehghan Baniani, D., et al. Prediction of transient pressure response in the petroleum reservoirs using orthogonal collocation. J. Pet. Sci. Eng. 2012, 98: 156-163.

Velez-Langs, O. Genetic algorithms in oil industry: An overview. J. Pet. Sci. Eng. 2005, 47(1-2): 15-22.

Zhang, J., Cheng, S., Zhu, C., et al. A numerical model to evaluate formation properties through pressure-transient analysis with alternate polymer flooding. Adv. GeoEnergy Res. 2019, 3(1): 94-103.

Zhao, K., Du, P. A new production prediction model for multistage fractured horizontal well in tight oil reservoirs. Adv. Geo-Energy Res. 2020, 4(2): 152-161. 